\title{
Cooperative Spin Caloritronic Devices
}

Jincheng $\mathrm{Lu}^{1}$, Fengjun Zhuo ${ }^{2}$, Zhouzhou Sun ${ }^{2 *}$ and Jianhua Jiang ${ }^{1^{*}}$

We report a concept of thermoelectric devices, cooperative spin caloritronics device (CSCD), where cooperation between two or more energy channels such as spin, charge and heat currents can significantly enhance energy efficiency of spin caloritronic devices. We derive the figure of merit and the maximum efficiency due to cooperative effect in analytic forms for a CSCD. Cooperative effects significantly improve the figure of merit and the maximum efficiency in spin caloritronic systems with multiple couplings effects. Several examples of CSCDs, including electrical and thermal current induced DW motion, spin-thermoelectric power generator and spin-thermoelectric cooling/heating, are studied to illustrate the usefulness of the cooperative effect. We compare the efficiency of CSCD with several recently proposed spin caloritronic devices. Our scheme provides a novel route to seek high performance materials and structures for future spin caloritronic devices.

Keywords: Thermoelectric effect; Thermoelectric devices; Cooperative effect

Received 3 October 2019, Accepted 16 December 2019

DOI: $10.30919 /$ esee 8 c353

\section{Introduction}

In the past few years, manipulation of magnetization and magnetic textures such as domain walls (DW) and skyrmions in ferromagnetic (FM) nanostructures has attracted a lot of attention because of fundamental interest and potential impacts on data storage devices and logic operations. ${ }^{1-5}$ Interplay between electronic spin, charge, and magnetization offers a promising physical mechanism for such manipulation. ${ }^{6}$ Especially, current-induced DW motion ${ }^{7-14}$ or skyrmions ${ }^{15-17}$ along highly conducting magnetic nanowires promises the development of novel spintronic devices with high density, performance and endurance at a very low cost per bit, such as racetrack memory. ${ }^{4}$ However, extensive experimental ${ }^{4,18}$ and theoretical $^{7-10}$ studies have shown that the critical current density to drive the motion of the conventional magnetic DW in FM nanostructures is on the order of $105-108 \mathrm{~A} \mathrm{~cm}^{2}$. Joule heating in such a high density information processing scheme becomes a serious issue because of the large current density which is necessary to overcome the pinning.

Recently it was proposed theoretically and verified experimentally that heat current can also serve as an efficient way to drive the motion of $\mathrm{DW}^{19-24}$ and skyrmions. ${ }^{25,26}$ It may be possible to exploit waste Joule heat to assist current-driven magnetic patterns motion for information processing. Alongside with electrical current and spin current, heat can be conducted to designated regions to achieve DW manipulation effectively. Such realizations lead to prosperous researches on spin caloritronics, ${ }^{27-29}$ an emerging field to study the interaction between spin, charge and heat currents, and magnetization in magnetic materials and structures. Pioneering researches have uncovered abundant physical mechanisms, such as electron-magnon, phonon-magnon, and charge-spin couplings that explain the versatile phenomena in spin caloritronics systems. ${ }^{30-33}$ Those couplings provide new ways to manipulate magnetic textures for information storage and processing. However, as for the situation of current-induced DW motion, energy efficiency in those couplings are still very low, which is one of the main challenges for spin-caloritronic applications. ${ }^{34-40}$

In this work, we propose a novel concept of cooperative spin caloritronics device (CSCD) where cooperation between two or more energy channels can significantly enhance energy

School of Physical Science and Technology \& Collaborative Innovation Center of Suzhou Nano Science and Technology, Soochow University, Suzhou 215006, China

${ }^{2}$ School of Physical Science and Technology \& Jiangsu Key Laboratory

of Thin Films, Soochow University, Suzhou 215006, China

*Email:phzzsun@suda.edu.cn (ZZS); jianhuajiang@suda.edu.cn (JHJ) 
efficiency of spin caloritronic devices. Theoretical foundation of such cooperative effects is established in Ref. [41] based on Onsager's theory of irreversible thermodynamics. A typical CSCD can be DW motion driven by coexisting electrical and heat currents. We show that cooperation between electrical and thermal currents induced DW motion can greatly improve the energy efficiency, surpassing the maximum achievable efficiency for DW motion induced solely by electrical or thermal current. Other CSCDs include spin-thermoelectric power generator and spin-thermoelectric cooling/heating. Our scheme provides a new route to significantly enhance energy efficiency and hence considerably reduce Joule heating for future advanced magnetic information storage and information processing.

\section{Basic Theoretical Framework}

Onsager's theory of irreversible thermodynamics establishes a general form to study nonequilibrium phenomena in thermodynamic systems. ${ }^{42-44}$ Like in classical systems with balanced friction and driving forces and moving in constant velocity, thermodynamic systems under external forces derives motions ("currents") at steady states. The relation between the forces $\vec{F}$ and currents $\vec{J}$ is generally written $\operatorname{as}^{45-48}$

$$
\vec{J}=\hat{M} \vec{F} \text { or } J_{n}=\sum_{k} M_{n k} F_{k},
$$

where the index $n(k)$ numerates all currents (forces), and $\hat{M}$ is called the Onsager matrix. When the forces are not too strong, the dependence of $\hat{M}$ on the forces can be ignored. Crosscorrelated responses (e. g., thermoelectric effect) allow conversion from the input energy to the output energy (e.g., thermal to electrical energy conversion). In general, a thermodynamic machine realizes its function via consuming the input energy and converting this energy into the output work/energy to achieve certain functionalities. According to the theory of irreversible thermodynamics, ${ }^{41-43,49}$ there are an equal number of forces and currents.

An important aspect of the performance of a machine is its energy efficiency. High energy-efficiency machine is demanded for future society not only to reduce energy cost, but also because damage of materials can be reduced if heating due to irreversible dissipation is reduced. It is hence crucial to improve the energy efficiency of functional materials and machines made of these materials. In practical applications, the first target is to find out the optimal energy efficiency and the condition that realizes the optimal energy efficiency for the functional materials/systems. ${ }^{48,50-60}$ A general theory was developed to fulfill this target for thermodynamic systems with arbitrary Onsager matrix (that may describe complex responses to multiple forces).

Each force $F_{n}$ has a conjugated current $J_{n}$ such that the reduction of total Gibbs free energy is given by

$$
-\dot{\mathrm{A}}_{t o t}=T \dot{S}_{t o t}=\sum_{n} J_{n} \mathrm{~F}_{n} \text {. }
$$

The reduction of the free energy $-\dot{\mathrm{A}}_{n}=J_{n} \mathrm{~F}_{n}$ is associated with the current $J_{n}$ and the force $\mathrm{F}_{n}$. Hence, the input and output free energy are

$$
-\dot{\mathrm{A}}_{\text {in }}=\sum_{n \in I} J_{n} \mathrm{~F}_{n}, \quad \dot{\mathrm{A}}_{\text {tot }}=\sum_{k \in O} J_{k} \mathrm{~F}_{k} .
$$

respectively. The symbols $I$ and $O$ in the above refer tothe free energy input and output, respectively. The output free energy is also the output work, i. e., $\dot{W}=\dot{A}_{\text {out }}$. For $\dot{A}_{\text {in }}>0$ the second-law energy efficiency is

$$
\phi=\frac{-\sum_{k \in O} J_{k} \mathrm{~F}_{k}}{\sum_{n \in I} J_{n} \mathrm{~F}_{n}}=\frac{\dot{\mathrm{A}}_{i n}-T \dot{S}_{t o t}}{\dot{\mathrm{A}}_{i n}} \leqslant 100 \% .
$$

Only in the reversible limit, $\dot{S}_{t o t} \rightarrow 0$, the above energy efficiency $\phi$ goes to its upper bound, $100 \%$.

\section{Maximum Energy Efficiency and Figure of Merit}

The maximum energy efficiency is obtained by solving the differential equation:

$$
\partial_{\vec{F}_{k}} \phi=0, \forall k \text {. }
$$

The current-force relation can be written in a compact form as

$$
\left(\begin{array}{c}
\vec{J}_{o} \\
\vec{J}_{I}
\end{array}\right)=\left(\begin{array}{cc}
\hat{M}_{O O} & \hat{M}_{O I} \\
\hat{M}_{I O} & \hat{M}_{I I}
\end{array}\right)\left(\begin{array}{c}
\vec{F}_{o} \\
\vec{F}_{I}
\end{array}\right) .
$$

The second-law energy efficiency is then given by

$$
\phi=\frac{-\vec{F}_{o}^{T} \vec{J}_{o}}{\vec{F}_{I}^{T} \vec{J}_{I}},
$$

where the symbols $O$ and $I$ are used to abbreviate the indices of forces and currents for energy output and input respectively, and the superscript $T$ stands for transpose of matrix and vector. From Eqs. (5), (6), and (7),

$$
\partial_{\vec{F}_{o}} \dot{\mathrm{A}}_{\text {out }}=\phi_{\max }\left(\partial_{\vec{F}_{o}} \dot{\mathrm{A}}_{\text {in }}\right) \text {, }
$$

which gives

$$
\vec{F}_{o}=-\frac{1+\phi_{\max }}{2} \hat{M}_{O O}^{-1} \hat{M}_{O I} \vec{F}_{l},
$$

where $\phi_{\max }$ is the maximal energy efficiency. $\hat{M}_{o O}^{-1}$ is welldefined as $\hat{M}_{o O}$ is a positive matrix. Inserting this into Eq. (6), we obtain

$$
\phi_{\max }=\frac{\frac{1}{4}\left(1-\phi_{\max }^{2}\right) \lambda}{1-\frac{1+\phi_{\max }}{2} \lambda},
$$

where $\lambda=\max \langle\hat{\Lambda}\rangle$ and $\langle\hat{\Lambda}\rangle=\vec{g}^{T} \hat{\Lambda} \vec{g}$. Here $\vec{g}$ is a normalized vector (i.e., $\vec{g}^{T} \vec{g}=1$ ) which is defined as 


$$
\vec{g}=\hat{M}_{I I}^{1 / 2} \vec{F}_{I} / \sqrt{\vec{F}_{I}^{T} \hat{M}_{I I} \vec{F}_{I}}
$$

Here

$$
\hat{\Lambda}=\hat{M}_{I I}^{-1 / 2} \hat{M}_{I O} \hat{M}_{o o}^{-1} \hat{M}_{O I} \hat{M}_{I I}^{-1 / 2} .
$$

Again, the inverse square root of the matrix $\hat{M}_{I I}$ is well defined since $\hat{M}_{I I}$ is a positive matrix. Eq. (10) is now a quadratic equation, which can be solved analytically. The physical solution with $\phi_{\max }<1$ is

$$
\phi_{\max }=\frac{\sqrt{\xi+1}-1}{\sqrt{\xi+1}+1}, \quad \xi=\frac{\lambda}{1-\lambda},
$$

where $\xi$ is the figure of merit and $\lambda$ is termed as the "degree of coupling". We denote the matrix $\hat{\Lambda}$ as the "coupling matrix". At the final stage, $\vec{F}_{I}$ or the normalized vector $\vec{g}$ is tuned to maximize $\langle\hat{\Lambda}\rangle$. The maximum value of $\langle\hat{\Lambda}\rangle$ is the largest eigenvalue of the matrix $\hat{\Lambda}$, i.e.,

$$
\lambda=\text { largest eigenvalue of } \hat{\Lambda} \text {. }
$$

It is proven in Ref. [41] that $\lambda \leqslant 1$ as bounded by the second law of thermodynamics. The limit $\lambda \rightarrow 1$ can be reached only in the reversible limit where the determinant of the Onsager matrix goes to zero.

For the same thermoelectric energy conversion device, which can function as a heat engine or a refrigerator, the figure of merit for the heat engine is the same as that for the refrigerator. This is because those two machines are related by reversed functions: the heat engine converts heat into electricity, whereas the refrigerator use the electricity to do cooling. This example can lead to quite general observations: a machine can function normally or reversed, leading to switched input and output. In the linear-response regime, one would expect that the largest eigenvalue of $\hat{M}_{I I}^{-1 / 2} \hat{M}_{I O} \hat{M}_{O O}^{-1} \hat{M}_{O I} \hat{M}_{I I}^{-1 / 2}$ is the same as the largest eigenvalue of $\hat{M}_{O O}^{-1 / 2} \hat{M}_{O I} \hat{M}_{I I}^{-1} \hat{M}_{I O} \hat{M}_{O O}^{-1 / 2}$ which has been proved in Ref. [41].

When the inverse of the Onsager matrix $\hat{M}^{-1}$ is readily available, the maximal efficiency can be obtained by differentiating over the currents as well, i.e., $\partial_{J_{k}} \phi=0, \forall k$. This approach gives the same results as in the above derivation but with $\hat{M}$ replaced by $\hat{M}^{-1}$, since the linear-response is now described by $\vec{F}=\hat{M}^{-1} \vec{J}$.

There are also other ways to express the figure of merit. For instance, we find that $(\hat{\imath}-\hat{\Lambda})^{-1}=\hat{M}_{I I}^{1 / 2}\left(\hat{M}^{-1}\right)_{I I} \hat{M}_{I I}^{1 / 2}$ because $\left(\hat{M}^{-1}\right)_{I I}=\left(\hat{M}_{I I}-\hat{M}_{I O} \hat{M}_{O O}^{-1} \hat{M}_{O I}\right)^{-1}$. We then obtain that

$$
\xi+1=\text { largest eigenvalue of } \hat{M}_{I I}^{1 / 2}\left(\hat{M}^{-1}\right)_{I I} \hat{M}_{I I}^{1 / 2} .
$$

Similarly, one can prove that $\xi+1=$ larg est eigenvalue of $\hat{M}_{o o}^{1 / 2}\left(\hat{M}^{-1}\right)_{O O} \hat{M}_{O O}^{1 / 2}$.

For systems with a single input (or output) channel (denoted as $k$ ), the above various expressions all give the same result as

$$
\xi+1=M_{k k}\left(\hat{M}^{-1}\right)_{k k}=\frac{M_{k k} m_{k k}}{\operatorname{det}(\hat{M})}
$$

where $m_{k k}$ and $\operatorname{det}(\hat{M})$ are the $(k, k)$-minor and the determinant of the Onsager matrix $\hat{M}$, respectively. For example, for systems of which the current-force relation is described by the following $3 \times 3$ symmetric Onsager matrix

$$
\hat{M}=\left(\begin{array}{lll}
M_{11} & M_{12} & M_{13} \\
M_{12} & M_{22} & M_{23} \\
M_{13} & M_{23} & M_{33}
\end{array}\right) .
$$

if $k=1$, then $m_{11}=M_{22} M_{33}-M_{23}^{2}$, and $\operatorname{det}(\hat{M})=M_{11} M_{22} M_{33}-$ $M_{13}^{2} M_{22}-M_{23}^{2} M_{11}-M_{12}^{2} M_{33}+2 M_{12} M_{13} M_{23}$. A neat way to express the results is to introduce the following dimensionless parameters

$$
q_{i j}=\frac{M_{i j}}{\sqrt{M_{i i} M_{i j}}} .
$$

The above coefficient represents the degree of coupling for energy conversion ${ }^{41}$ between the $i^{- \text {th }}$ channel and the $j^{- \text {th }}$ channel. The second law of thermodynamics requires that $\left|q_{i j}\right| \leqslant 1$. One can show that the Onsager matrix can be expressed using $q_{i j}$ if the following transformation is introduced, $F_{i} \rightarrow F_{i} \sqrt{M_{i i}}$ and $J_{i} \rightarrow J_{i} \sqrt{M_{i i}}$. The cooperative figure of merit, given in (16) for systems with $3 \times 3$ symmetric Onsager matrix, is then expressed neatly as

$$
\xi=\frac{1-q_{23}^{2}}{1-q_{12}^{2}-q_{13}^{2}-q_{23}^{2}+2 q_{12} q_{13} q_{23}}-1 .
$$

\section{Electrical and Thermal Current Induced DW Motion}

It has been shown that electrical and thermal current induced DW motion in a magnetic nanowire (Fig. 1(a)) can be described by a phenomenological linear-response equation $\vec{F}=\hat{M} \vec{I},{ }^{20,63}$ where

$$
\begin{gathered}
\vec{J}=\left(J_{c}, J_{Q}, J_{w},\right)^{T}, \\
\vec{F}=\left(\Delta V, \Delta T / T, 2 A M_{s} H_{\text {ext }}\right)^{T} .
\end{gathered}
$$

and

$$
\hat{M}=\left(\begin{array}{lll}
M_{c c} & M_{c Q} & M_{c w} \\
M_{c Q} & M_{Q Q} & M_{Q w} \\
M_{c w} & M_{Q w} & M_{w w}
\end{array}\right) .
$$

The three thermodynamic currents are the electrical current $J_{c}$, the thermal current $J_{Q}$, and the velocity of DW motion $I_{w}=\dot{r}_{w}$ where $r_{w}$ is the center of the DW. The three thermodynamic forces that induce the currents are the voltage $\Delta V=\left(\mu_{h}-\mu_{c}\right) / e$ with $\mu_{h}\left(\mu_{c}\right)$ being the 
electrochemical potential of the hot (cold) terminal, the temperature difference $\Delta \mathrm{T} / \mathrm{T}=\left(T_{h}-T_{c}\right) / T$ with $T_{h}\left(T_{c}\right)$ being the temperature of the hot (cold) terminal, and the external magnetic field $H_{e x t}$. Following Ref. [63], the coefficients of thelinear-response matrix can be written as follows: $M_{c c}=R, M_{Q Q}=R / L T^{2}, \quad M_{w w}=\frac{2 \mu_{0} \alpha A M_{s}}{\Delta \gamma}, \quad M_{c Q}=$ $S R / L T, M_{c w}=\hbar p \beta / e \Delta$ and $M_{w Q}=\frac{\hbar}{e} \frac{1}{\Delta L T}\left(S^{\prime} \beta^{\prime}-S p \beta\right)$. Here $R=l / \sigma A$ is electrical resistance of the device where $\sigma$ is the electrical conductivity, $l$ and $A$ are the length and area of the device, respectively. For a nanowire system as illustrated in Fig. 1, we take $l=1 \mu \mathrm{m}$ and $A=100 \mathrm{~nm}^{2}$ for our calculation. $L=2.443 \times 10^{-8} \mathrm{~W} \Omega \mathrm{K}^{-2}$ is the Lorenz number for metals. $T=300 \mathrm{~K}$ is the room temperature. We chose the material parameters that areclose to those of the permalloy, viz., thesaturation magnetization $M_{s}=860 \times$ $10^{3} \mathrm{~A} / \mathrm{m}$, the DW width $\Delta=100 \mathrm{~nm}$, the Gilbert damping $\alpha=0.01$, the electrical conductivity $\sigma=10^{5}(\Omega \mathrm{cm})^{-1}$, and the Seebeck coefficient $S=100 \mu \mathrm{V} / \mathrm{K}$. $\mu_{0}$ is the vacuum permeability, $e$ is the electron charge, and $\gamma$ is the gyromagnetic ratio. Microscopically, the spin polarization, the Seebeck coefficient and the spin Seebeck coefficient are given by $P=\left\langle s_{z}\right\rangle, S=\frac{\langle E\rangle}{e T}$ and $S^{\prime}=\frac{\left\langle E s_{z}\right\rangle}{e T}$.
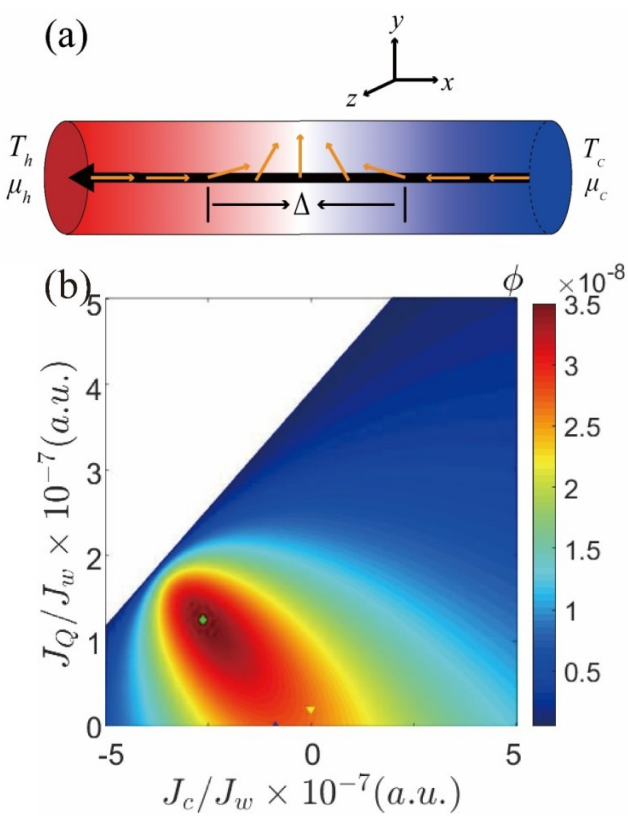

Fig. 1 (Color online) (a) Schematic of electrical and thermal currents induced a 1D head-to-head DW motion in a ferromagnetic nanowire. (b) The energy efficiency, $\phi$, as a function of the ratio of the input currents and output current, $J_{c} / J_{w}$ and $J_{Q} / J_{w}$. The parameters are $P=0.5, S=100 \mu \mathrm{V} / \mathrm{K}$ and $S^{\prime}=-80 \mu \mathrm{V} / \mathrm{K}$. The device does not work as currentdriven DW motion function in the white region.
Note that, throughout this paper, we have set the energy zero to be the(equilibrium) chemical potential, i. e., $\mu \equiv 0, s_{z}=1$ or -1 for spin up and down, respectively. The average here is defined as $\langle O\rangle=\sigma^{-1} \int d E\left(-\frac{\partial n_{F}}{\partial E}\right) \sum_{s} \sigma^{(s)}(E) O$, where $\sigma^{(s)}(E)(s=\uparrow, \downarrow)$ is the spin- and energy-dependent conductivity. $\sigma=\int d E\left(-\frac{\partial n_{F}}{\partial E}\right) \sum \sigma^{(s)}(E)$ is the electrical conductivity. $\mathrm{n}_{F}=1 /\left[\exp \left(\frac{E}{K_{B} T}\right)+1\right]$ is the Fermi distribution of the carrier. The relationships presented here are the generalized Mott relations forspin-caloritronic systems. The $\beta$ and $\beta^{\prime}$ terms are regarded as crucial in understanding magnetic DW dynamics driven by electrical and thermal currents. ${ }^{20}$ Although $\beta$ and $\beta^{\prime}$ can generally be different, in the following estimation we will take $\beta=\beta^{\prime}=0.1$.

The maximum efficiency is determined by the figure of merit and the degree of coupling according to Eq. (13). From Eq. (18), $q_{c Q}$ represents the degree of coupling between electrical and thermal energy. The figure of merit for electrical current-induced DW motion is $\xi_{c w}=\frac{q_{c w}^{2}}{1-q_{c w}^{2}}$, and that for thermal current-induced DW motion is $\xi_{Q_{w}}=$ $\frac{q_{Q_{w}}^{2}}{1-q_{Q_{w}}^{2}}$. The second law of thermodynamics requires that $\left|q_{i j}\right| \leqslant 1$, so that the maximum efficiency is bounded from above to ensure $\phi_{\max } \leqslant 100 \%$. The figure of merit of the cooperative DW motion induced by the concurrentelectrical and thermal currents is

$$
\xi=\frac{1-q_{c Q}^{2}}{1-q_{c w}^{2}-q_{Q_{w}}^{2}-q_{c Q}^{2}+2 q_{c w} q_{Q_{w}} q_{c Q}}-1,
$$

which determines the maximum energy efficiency through Eq. (13). It can be proved that the cooperative figure of merit $\xi$ is always larger than (or, at least, equal to) $\xi_{c w}$ and $\xi_{Q w}$ (see App. C). This is because the maximum efficiency given by the figure of merit in Eq. (18) is the global maximum of the efficiency, while $\xi_{c w}$ and $\xi_{Q w}$ only give the (conditional) maximum efficiency without heat or electrical current, respectively.

Fig. 1(b) demonstrates the energy efficiency as a function of the electrical and heat currents for a typical case. Specifically, the energy efficiency, $\phi$, as a function of the ratio of the input currents to the output current, $J_{c} / J_{w}$ and $J_{Q} / J_{w}$, is plotted. We set $p=0.5, S=100 \mu \mathrm{V} / \mathrm{K}$ and $S^{\prime}=$ $-80 \mu \mathrm{V} / \mathrm{K}$. These two currents can be of the same sign, or the opposite sign depending on the directions of the electrochemical potential gradient and the temperature gradient. Here we choose negative temperature gradient (along the $x$ direction) and vary the direction of the 
electrochemical potential gradient. The down-triangle (uptriangle) point represents the maximal energy efficiency for the magnetic DW motion driven solely by the thermal (electrical) current. The rhombus point represents the global maximum efficiency for the magnetic DW motion induced by the concurrent electrical and thermal currents. The cooperative effect is clearly manifested by the fact that the global maximum efficiency is much greater than the optimal efficiency's for the DW motion driven by only one of the currents, electrical or thermal current.

The enhancement of the maximum energy efficiency due to cooperative effects, measured by $\frac{\phi_{\max }}{\max \left(\phi_{c w}, \phi_{Q w}\right)}$, as a function of $P$ and $S^{\prime}$ is plotted in Fig. 2(a). The energy efficiency is significantly improved by the cooperative effect when $S^{\prime} /(100 \mu \mathrm{V} / \mathrm{K})$ is approximately negative twice of the spin polarization $P$. Fig. 2(b) shows the enhancement of the maximum energy efficiency as a functionof the thermoelectric coupling coefficient $q_{c Q}=S / \sqrt{L}$ when the electrical and thermal current-induced DW motion coefficients, $q_{c u}$ and $q_{Q w}$, aresetas constants (i.e., $S^{\prime} \beta^{\prime}-S p \beta$ is fixed to be constant). Counterintuitively, although the thermoelectric coupling coefficient $q_{c Q}$ has nothing to do with the optimal energy efficiency of the electrical (or thermal) current induced DW motion, it has strong effects on the maximum efficiency for the magnetic DW motion driven by coexisting electrical and thermal currents. As already manifested in Eq. (22), the global maximum efficiency depends on the thermoelectric coupling coefficient $q_{c Q}$. Hence tuning $q_{c Q}$ can help improving the maximum efficiency. Fig. 2(b) shows that the dependence of the efficiency enhancement factor, $\frac{\phi_{\max }}{\max \left(\phi_{c w}, \phi_{Q_{w}}\right)}$, onthe thermoelectric coupling coefficient $q_{c Q}$ is not monotonic.

The non-monotonic behavior of the enhancement factor, $\frac{\phi_{\max }}{\max \left(\phi_{c w}, \phi_{Q_{w}}\right)}$, can be understood via Eq. (22), since the optimal efficiency has a one-to-one correspondence to the figure of merit. We emphasize two important aspects of the cooperative effect. First, the magnetic DW motion induced by the electrical and the thermal currents can be of the same direction, leading to constructive interplay between the two driving factors. If their directions are opposite, however, there will be destructive interplay between the two. Second, entropy production that limits the maximum efficiency, has contribution from all processes, including electricity and heat to magnetic energy conversion, as well as the conversion between electricity and heat energy.

Tuning the $q_{c Q}$ modifies the entropy production associated with the energy conversion between electricity and heat, and hence affects the maximum energy efficiency. However, the cooperative maximum efficiency is always greater than or equal to the maximum efficiency for magnetic DW motion driven by the electrical current or the thermal current [See App. C]. Therefore, unfavorable values of $q_{c Q}$ can only reduce the enhancement factor down to 1 , which is realized only when $q_{c \ell}=\frac{q_{c w}}{q_{Q w}}$ or $q_{c Q}=\frac{q_{Q_{w}}}{q_{c w}}$. For the parameters chosen in Fig. 2(b), the enhancement factor has
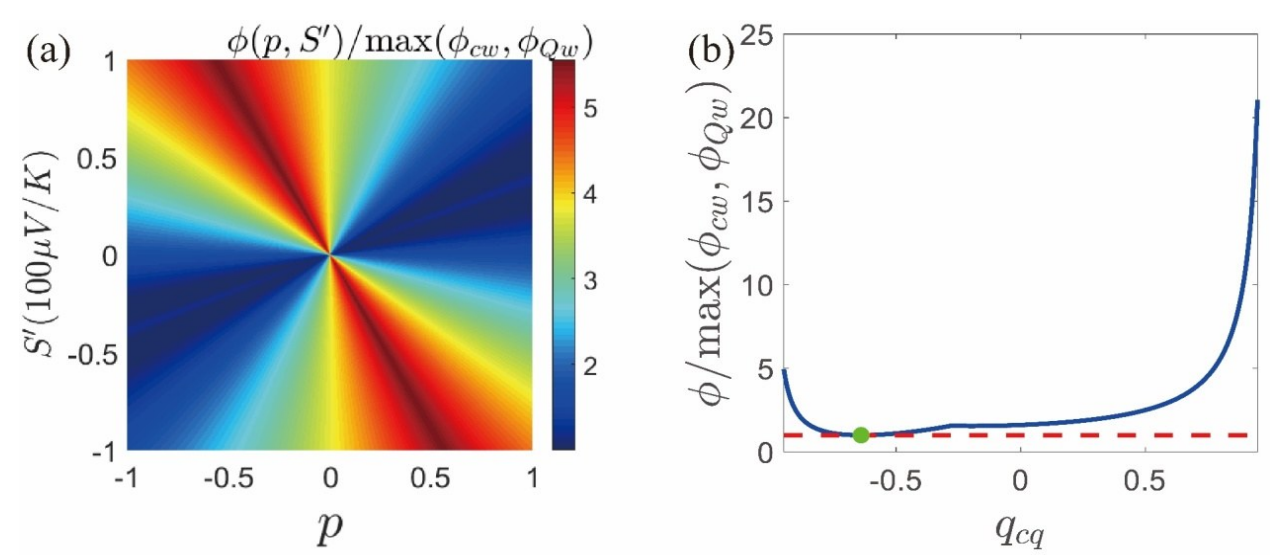

Fig. 2 (Color online) (a) The enhancement factor of the maximum energy efficiency due to the cooperative effect, $\frac{\phi}{\max \left(\phi_{c w}, \phi_{Q_{w}}\right)}$, as a function of $P$ and $S^{\prime}$. (b) The enhancement factor of the maximum energy efficiency as a function of the thermoelectric coupling coefficient $q_{c Q}$. The red dashed line represents the condition with $\frac{\phi}{\max \left(\phi_{c w}, \phi_{Q_{w}}\right)}=1$, which is reached at the green triangle point. 
a minimum when $q_{c Q}=\frac{q_{Q_{w}}}{q_{c w}}$ as illustrated by the triangle point in Fig. 2(b)]. Away from this point, the cooperative effect can considerably enhance the optimal energy efficiency. This gives rise to a useful route toward high energy-efficiency: tuning the thermoelectric coupling $q_{c Q}$ to enhance the cooperative effect for high energy-efficiency. Note that this method can be applied to materials with low energy-efficiency for the electrical (or thermal) drivingmagnetic DW motion, which might be of practical usage. We would also emphasize that although the electrical and thermal currents coexist in most practical situations, the cooperative maximum efficiency can be reached only by properly tuning the temperature and electrochemical potential gradients, as shown in Fig. 1(b).
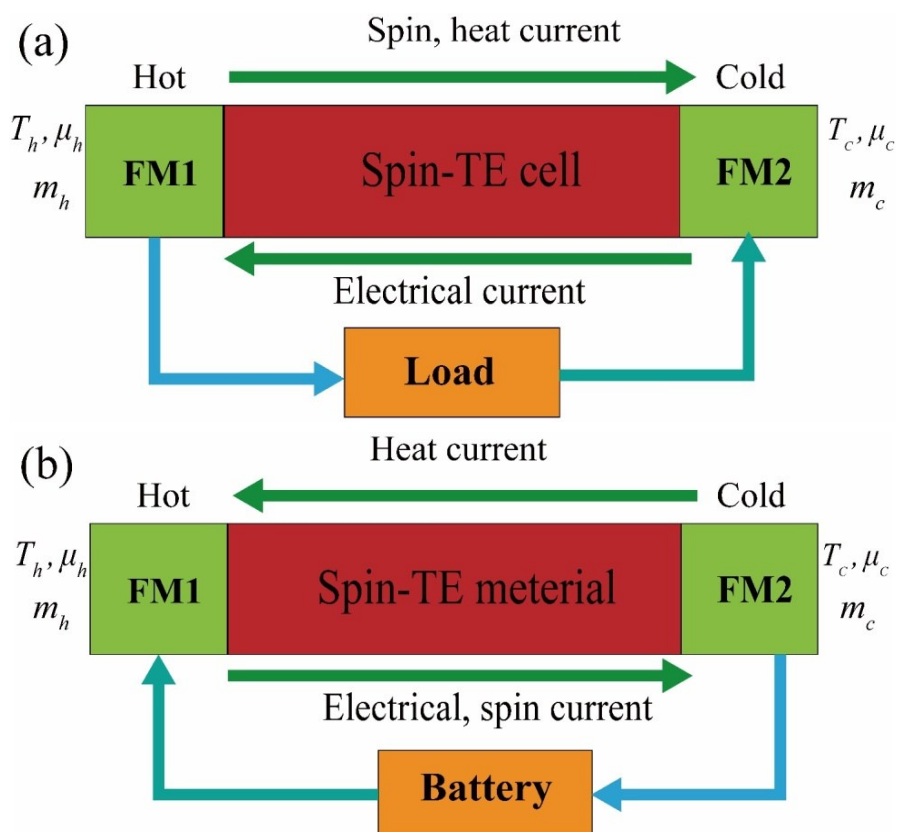

Fig. 3 (Color online) Schematic of (a) the spin-thermoelectric powergenerator and (b) the spin-thermoelectric cooling/heatpumper. Aspin-thermoelectric ("spin-TE") material (i. e., a conducting magneticmaterial) sandwiched between two ferromagnetic (FM) electrodes withdifferent temperature, $T_{h}>T_{c}$ where the subscripts $h$ and $c$ denotingthe hot and cold terminals, respectively.

\section{Cooperative Effects in Spin-Thermoelectric Systems.}

In a magnetic materialthe coupled spin, charge and heat transport is described by the following phenomenological equation, ${ }^{27,36}$

$$
\left(\begin{array}{l}
J_{c} \\
J_{s} \\
J_{\ell}
\end{array}\right)=\left(\begin{array}{ccc}
G & G P & G S T \\
G P & G & G S^{\prime} T \\
G S T & G S^{\prime} T & K_{0} T
\end{array}\right)\left(\begin{array}{c}
\Delta V \\
\Delta m \\
\Delta T / T
\end{array}\right) .
$$

where $J_{c}=j^{(\uparrow)}+j^{(\downarrow)}, I_{s}=j^{(\uparrow)}-j^{(\downarrow)}$ with $j^{(\uparrow)}$ and $j^{(\downarrow)}$ denoting the electrical currents of the spin-up andspin-down electrons, respectively. $\Delta V=\left(\mu_{h}-\mu_{c}\right) / e$ is the voltage bias due to the electrochemical potential difference between the hot and cold terminals. Here, the subscripts $h$ and $c$ denote the hot and cold terminals, respectively. $\mu \equiv\left(\mu_{\uparrow}+\mu_{\downarrow}\right) / 2$ is the charge electrochemical potential, while $\Delta m \equiv\left(\mu_{\uparrow}-\mu_{\downarrow}\right) /(2 e)$ is the spin chemical potential. Here, $\mu_{\uparrow}$ and $\mu_{\downarrow}$ are the electrochemical potentials for spin-up and spin-down electrons, respectively. $\Delta m=m_{h}-m_{c}$ is the difference of the spin chemical potential across the device. $G=\sigma A / l$ is the electrical conductance and $K_{0}=\kappa_{0} A / l$ is the heat conductance of the device at $\Delta V=\Delta m=0$ with $\kappa_{0}$ being the heat conductivity. Possible applications of the system include electrical power generator, cooling/heatpumping, and spin pumper (the former two are illustrated in Fig. 3).

We first discuss the spin-thermoelectric power generator driven bythe coexisting temperature gradient $\Delta T / T$ and spin chemical potential bias $\Delta m$ [Fig. 3(a)]. The energy efficiency is given by $\phi=-J_{c} \Delta V /\left(J_{Q} \Delta T / T+J_{s} \Delta m\right)$. Using Eqs. (13) and (23) we obtain

$$
\xi=\frac{\kappa_{0} P^{2}+\sigma T\left(S^{2}-2 P S S^{\prime}\right)}{\kappa_{0}\left(1-P^{2}\right)-\sigma T\left(S^{2}-2 P S S^{\prime}+S^{\prime 2}\right)} .
$$

Again, the above figure of merit is always greater than (or, at least, equal to) both the figure of merit forthermoelectric power generator $\xi_{T E}=\frac{\sigma S^{2} T}{\kappa_{0}-\sigma S^{2} T}$ and the figure of merit for spin-charge conversion

$$
\xi_{S E}=\frac{P^{2}}{1-P^{2}}
$$

(see App. C).

We show in Fig. 4(a) that the enhancement factor of the figure ofmerit induced by the cooperative effect is considerable when $P$ and $S^{\prime}(100 \mu \mathrm{V} / \mathrm{K})$ differs from each other (especially when they have different signs). It is shown in the App. A that the transport coefficients are bounded by the second-law of thermodynamics. ${ }^{64}$ The white regions in Fig. 4 are forbidden by the second-law of thermodynamics. The cooperative figure of merit $\xi$ can be very large for large $P$ and $S^{\prime}$ as shown in Fig. 4(b). Particularly, the figure of merit $\xi$ is very large near the boundary of the allowed region. Exactly speaking the boundary represents the limitwhen the determinant of Onsager matrixbecomes zero $\left[\begin{array}{lll}i . & \text { e., } \quad \kappa_{0}\left(1-P^{2}\right)-\end{array}\right.$ $\left.\sigma T\left(S^{2}-2 P S S^{\prime}+S^{\prime 2}\right)=0\right]$. Thus, the maximum energy efficiency approaches its upper bound, $100 \%$, and the figure of merit $\xi$ goes to infinityin approaching the boundary of the allowed region in Fig. 4 (The divergent behavior of $\xi$ 
cannot be resolved in the figure due to restricted data range and resolution). We also plot the enhancement factor as a function of $P$ and the thermoelectric degree of coupling, $\lambda_{T E}=\frac{\sigma S^{2} T}{\kappa_{0}}$, for $S^{\prime}=25 \mu \mathrm{V} / \mathrm{K}$ in Fig. 4(c). From Fig. 4(c) the enhancement of energy efficiency due to cooperative effects is considerably large for large $|P|$ and $\lambda_{T E}$. The enhancement factor as a function of the spin-Seebeck coefficient $S^{\prime}$ and the thermoelectric degreeof coupling $\lambda_{T E}$ for $P=0.5$ is plotted in Fig. 4(d). We found that the enhancement is considerable for negative $S^{\prime}$ with large $\left|S^{\prime}\right|$. That is, strong enhancement can beobtained when $S^{\prime}<0$ for moderate and small thermoelectric degree of coupling $\lambda_{T E}$. The negative $S^{\prime}$ and positive $S$ require that the Seebeck coefficient of the minority-spin carriers to be negative (with large absolute value) while the majority-spin carriers have positive Seebeck coefficient.

We now consider the spin-thermoelectric cooling/ heating driven by the coexisting voltage $\Delta V$ and spin chemical potential bias $\Delta m$. The coefficient of performance of the refrigerator (and heat pumper) is defined as
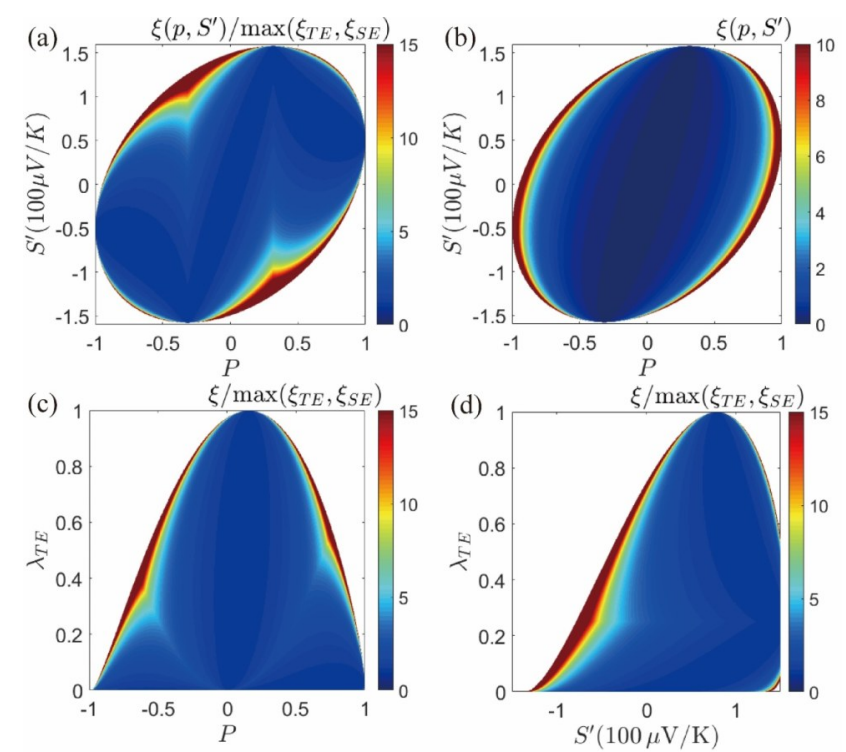

Fig. 4 (Color online) Spin-thermoelectric power generation.

(a) The enhancement factor of the figure of merit due to cooperative effects, $\frac{\xi}{\max \left(\xi_{T E}, \xi_{S E}\right)}$, as a function of $P$ and $S^{\prime}$.

The parameters are $S=50 \mu \mathrm{V} / \mathrm{K}$ and $T=300 \mathrm{~K}$. The heat conductivityis $\kappa_{0}=\sigma L T$ with the Lorenz number of $L=$ $2.5 \times 10^{-8} \mathrm{~W} \Omega \mathrm{K}^{-2}$. (b) The cooperative figure of merit $\xi$ as a function of $P$ and $S^{\prime}$. (c) The enhancement factor of the figure of merit as a function of $P$ and $\lambda_{T E}=\frac{\sigma S^{2} T}{\kappa_{0}}$ for $P=$ 0.5. In all of the above figures, the white regions are forbidden by the second-law ofthermodynamics.

$$
\eta \equiv \frac{\dot{Q}}{\dot{W}}=\frac{T}{\Delta T} \frac{-J_{Q} \Delta T / T}{J_{c} \Delta V+J_{s} \Delta m}=\eta_{c} \phi
$$

where $\eta_{c}=\frac{T}{\Delta T}$ is the Carnot efficiency. The schematic of spin-thermoelectric cooling/heating is shown in Fig. 3(b) and here we discuss cooling as an example. Using Eqs. (13) and (23), we obtain

$$
\xi=\frac{\sigma T\left(S^{2}-2 P S S^{\prime}+S^{\prime 2}\right)}{\kappa_{0}\left(1-P^{2}\right)-\sigma T\left(S^{2}-2 P S S^{\prime}+S^{\prime 2}\right)} .
$$

The above figure of merit is greater or equal to both the figure of merit for thermoelectriccooling, $\xi_{T E}$, and the figure of merit for the spin-Peltier cooling

$$
\xi_{S P}=\frac{\sigma T S^{\prime 2}}{\kappa_{0}-\sigma T S^{\prime 2}} .
$$

The enhancement factor of the figure of merit induced by cooperative effect is plotted in a wide parameter range in Fig. 5(a). The figure of merit is significantly improvedby cooperative effect when $P$ strongly deviates from $S^{\prime} /(100 \mu \mathrm{V} / \mathrm{K}$ ) (particularly when the two have opposite signs). From Fig. 5(b), one can see that the cooperative
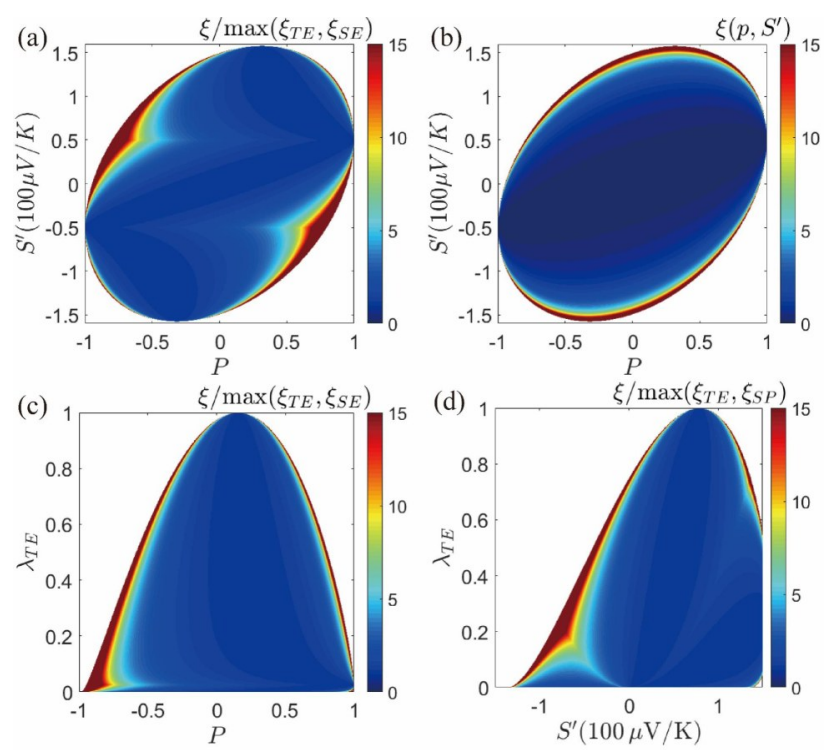

Fig. 5 (Color online) Spin-thermoelectric cooling. (a) The enhancement factor of the figure of merit due to cooperative effects, $\frac{\xi}{\max \left(\xi_{T E}, \xi_{S P}\right)}$, as a function of $P$ and $S^{\prime}$. The parameters are $S=50 \mu V / K$ and $\mathrm{T}=300 \mathrm{~K}$. The heat conductivity is $\kappa_{0}=\sigma L T$ with the Lorenz number of $L=2.5 \times 10^{-8} \mathrm{~W}_{\Omega} \mathrm{K}^{-2}$. (b) The cooperative figure of merit $\xi$ as a function of $P$ and $S^{\prime}$. (c) The enhancement factor of the figure of merit as a function of $P$ and $\lambda_{T E}=\frac{\sigma S^{2} T}{\kappa_{0}}$ for $P=0.5$. In all ofthe above figures, the white regions are forbidden by the second-law ofthermodynamics. 
figure of meritcan be much larger than 1 when $P$ and $S^{\prime} /(100 \mu \mathrm{V} / \mathrm{K})$ are sufficiently different. In such a regime, the spin-thermoelectric refrigeration is much more efficient than the thermoelectric cooling in the same material. Particularly, when $S^{\prime} /(100 \mu \mathrm{V} / \mathrm{K})$ is close to -0.5 , the spinthermoelectric refrigeration becomes more efficient than the thermoelectric refrigeration. Figs. 5(c) and 5(d) indicate that the enhancement of figure of merit is strong when $P$ and $S^{\prime} /(100 \mu \mathrm{V} / \mathrm{K})$ are very different, as shown by Figs. 5(b) and $5(\mathrm{~d})$.

\section{Thermal, Electrical and Mechanical Inter-Coupling in A Magnetic Wire}

In general the Onsager matrix may not be symmetric. A simple case when it is anti-symmetric. That is, $M_{i j}=-M_{j i}$, $\forall i \neq j$. Note that $\vec{F}_{o}^{T} \hat{M}_{o 0} \vec{F}_{o}=\vec{F}_{o}^{T} \hat{\bar{M}}_{o o} \vec{F}_{o}$, where $\hat{\bar{M}}_{o O}=$ $\left(\hat{M}_{o O}+\hat{M}_{o o}^{T}\right) / 2$. Similarly, $\hat{M}_{I I}$ can be replaced by $\hat{\bar{M}}_{I I}=$ $\left(\hat{M}_{I I}+\hat{M}_{I I}^{T}\right) / 2$. Then we have

$$
\left(\begin{array}{c}
\vec{I}_{o} \\
\vec{I}_{I}
\end{array}\right)=\left(\begin{array}{cc}
\hat{\bar{M}}_{O O} & \hat{M}_{O I} \\
\hat{M}_{O I}^{T} & \hat{\bar{M}}_{I I}
\end{array}\right)\left(\begin{array}{c}
\vec{F}_{o} \\
\vec{F}_{I}
\end{array}\right) .
$$

Interestingly we note that the Onsager matrix can be symmetrized in the following representation

$$
\left(\begin{array}{c}
\vec{I}_{O} \\
\vec{F}_{I}
\end{array}\right)=\left(\begin{array}{cc}
\hat{L}_{O O} & \hat{L}_{O I} \\
\hat{L}_{O I}^{T} & \hat{L}_{I I}
\end{array}\right)\left(\begin{array}{c}
\vec{F}_{o} \\
\vec{I}_{I}
\end{array}\right),
$$

where

$$
\begin{gathered}
\hat{L}_{O O}=\hat{\bar{M}}_{O O}-\hat{M}_{O I} \hat{\bar{M}}_{I I}^{-1} \hat{M}_{I O}, \\
\hat{L}_{O I}=\hat{M}_{O I} \hat{\bar{M}}_{I I}^{-1}, \\
\hat{L}_{I O}=-\hat{\bar{M}}_{I I}^{-1} \hat{M}_{I O}, \\
\hat{L}_{I I}=\hat{\bar{M}}_{I I}^{-1} .
\end{gathered}
$$

It can be shown easily that $\hat{L}_{I 0}=\hat{L}_{O I}$ since $\hat{M}_{I 0}=-\hat{M}_{O I}^{T}$. Furthermore, we notice that $\vec{F}_{O}$ and $\vec{I}_{I}$ are independent of each other, and the optimal efficiency is reached at

$$
\partial_{\vec{F}_{0}} \phi=0, \quad \partial_{\vec{I}_{I}} \phi=0 .
$$

Consequently the degree of coupling can be expressed as the largest eigenvalue of the following coupling matrix

$$
\hat{\Lambda}=\hat{L}_{I I}^{-1 / 2} \hat{L}_{I O} \hat{L}_{O O}^{-1} \hat{L}_{O I} \hat{L}_{I I}^{-1 / 2}
$$

More generally, if $M_{i j}=-M_{i j}, \forall i \in 1$ and $\forall j \in 2$, but $M_{i j}=M_{j i}$ if $i, j \in 1$ or $i, j \in 2$ [Onsager matrix with such properties describes systems where the forces $F_{i}(i \in 1)$ are even under time-reversal but $F_{j}(j \in 2)$ are odd, or vice versa], the Onsager matrix can be symmetrized similarly with

$$
\begin{gathered}
\hat{L}_{11}=\hat{M}_{11}-\hat{M}_{12} \hat{M}_{22}^{-1} \hat{M}_{21}, \\
\hat{L}_{12}=\hat{M}_{12} \hat{M}_{22}^{-1}, \\
\hat{L}_{21}=-\hat{M}_{22}^{-1} \hat{M}_{21}, \\
\hat{L}_{22}=\hat{M}_{22}^{-1} .
\end{gathered}
$$

After such transformation, the Onsager matrix becomes symmetric and Eq. (33) can be applied directly in calculating the optimal efficiency. The new response equationis then

$$
\left(\begin{array}{l}
\vec{I}_{1} \\
\vec{F}_{2}
\end{array}\right)=\left(\begin{array}{ll}
\hat{L}_{11} & \hat{L}_{12} \\
\hat{L}_{12}^{T} & \hat{L}_{22}
\end{array}\right)\left(\begin{array}{l}
\vec{F}_{1} \\
\vec{I}_{2}
\end{array}\right) .
$$

It has been shown in Ref. [63] that a magnetic nanowire contains a transverse DW [Fig. 1(a)], the wire is mounted in a low-friction bearing such that it can rotate aroundthe $x$ axis. A mechanical torque $\tau_{\text {ext }}^{\text {mech }}$ can also be applied to it. The system is driven by an applied magneticfield $H_{\text {ext }}$, the voltage bias $\Delta V$ and the temperature bias $\Delta T$. The electrical, thermal, magnetic and mechanicalresponses of the magnetic wire can be described by the following phenomenological linear-response equation

$$
\vec{I}=\hat{M} \vec{F},
$$

where the currents and forces are

$$
\begin{gathered}
\vec{I}=\left(\vec{J}_{c}, \vec{J}_{Q}, \vec{J}_{w}, \dot{\varphi}\right)^{T}, \\
\vec{F}=\left(\Delta V, \Delta T / T, 2 A M_{s} H_{\text {ext }} \tau_{\text {ext }}^{\text {mech }}\right)^{T},
\end{gathered}
$$

respectively. The response matrix is

$$
\hat{M}=\left(\begin{array}{cccc}
M_{c c} & M_{c Q} & M_{c w} & M_{c \varphi} \\
M_{c Q} & M_{Q Q} & M_{Q w} & M_{Q \varphi} \\
M_{c w} & M_{Q_{w}} & M_{w w} & M_{w \varphi} \\
-M_{c \varphi} & -M_{Q \varphi} & -M_{w \varphi} & M_{\varphi \varphi}
\end{array}\right) .
$$

In the above Onsager matrix the off-diagonal terms in the last row have opposite sign of those in the last column. We can apply the technique developed in the above section to symmetrize the Onsager matrix. If we denotet he indices $(c, Q, w)$ as 1 and the index $\varphi$ as 2 , we will have the response equation in the form of

$$
\left(\begin{array}{l}
\vec{F}_{1} \\
\vec{I}_{2}
\end{array}\right)=\left(\begin{array}{ll}
\hat{L}_{11} & \hat{L}_{12}^{T} \\
\hat{L}_{12} & \hat{L}_{22}
\end{array}\right)\left(\begin{array}{l}
\vec{I}_{1} \\
\vec{F}_{2}
\end{array}\right),
$$

where the matrix $\hat{L}$ is obtained from Eq. (34). After that the figure of merit and degree of coupling can be obtained by using Eqs. (13) and (14).

We now shall input the numbers of transport coefficients given in Ref. [63]. We shall use the inverse Onsager matrix and covert it into the dimensionless coefficients $q_{i j}$ given in Eq. (18). From the transport coefficients in Ref. [63], we obtain the following dimensionless parameters,

$$
\begin{gathered}
q_{i j}=\frac{M_{i j}}{\sqrt{M_{i i} M_{i j}}}, \forall i, j, \quad q_{i j}=1, \forall i \\
q_{c Q}=0.255, \quad q_{c \varphi}=-0.0218, q_{c w}=0.066, \\
q_{Q \varphi}=0, \quad q_{Q w}=0, \quad q_{w \varphi}=-0.330 .
\end{gathered}
$$

For the symmetrized matrix 


$$
\begin{gathered}
\tilde{q}_{i j}=\frac{L_{i j}}{\sqrt{L_{i i} L_{j j}}} \forall i, j, \quad \tilde{q}_{i j}=1, \forall i \\
\tilde{q}_{c Q}=0.255, \quad \tilde{q}_{c \varphi}=-0.0218, \quad \tilde{q}_{c w}=0.0695, \\
\tilde{q}_{Q \varphi}=0, \quad \tilde{q}_{Q w}=0, \quad \tilde{q}_{w \varphi}=-0.313 .
\end{gathered}
$$

From the above data, we find that the figure of merit fora pair of energy channels,

$$
\begin{gathered}
\xi_{c Q}=0.0695, \quad \xi_{c \varphi}=0.000475, \quad \xi_{c w}=0.00485, \\
\xi_{Q \varphi}=0, \quad \xi_{Q w}=0, \quad \xi_{w \varphi}=0.109 .
\end{gathered}
$$

We shall demonstrate the cooperative effect by considering moving the magnetic domain with (i) both electrical and heat forces (ii) all the other forces (i. e., electrical, heat, and mechanical forces).

For (i) we parameterize the electrical and heat currentsas

$$
J_{c}\left(k_{B} T\right) / e=j_{0} \cos (\theta), J_{Q}=j_{0} \sin (\theta),
$$

where $j_{0}=\sqrt{J_{c}^{2}\left(k_{B} T\right)^{2} / e^{2}+J_{Q}^{2}}$ is the total magnitudeof the currents. The figure of merit as the function of theangle $\theta$ is plotted in Fig. 6(a). It is seen that, although heat current cannot induce the motion of magnetic domain, there still emerge a cooperative effect when both heat and electrical currents are used together. If we lookclosely at the degree of coupling for a given $\theta$,

$$
\lambda=\frac{\tilde{q}_{c w}^{2} \cos ^{2}(\theta)}{1+\tilde{q}_{c Q}^{2} \sin ^{2}(\theta)} .
$$

Although the numerator is reduced as part of the total current, the heat current, does not couple to the magnetic domain motion, the denominator is also reducedat some range of $\theta$ due to correlation between heat and electrical currents. Such correlation reduces entropy production and enhances the efficiency when $\sin (2 \theta)<0$. This becomes particularly clear in Fig. 6(b) where the output work $\dot{W}$ and the total entropy production $\dot{S}_{\text {tot }}$ are plotted against $\theta$. It is seen that the output work is maximum when $\theta=0$ or $\pi$. Going away from $\theta=\pi$ into the region $3 \pi / 4<\theta<\pi$ will reduce the output work as well as the total entropy production. The latter is more prominent which yields an increase of the efficiency.

Cooperative effect also manifest when more forces areinvolved. For case (ii), domain motion is driven by electrical, heat, and mechanical motions. We can parameterize the input currents as follows,

$$
\begin{gathered}
J_{c} / e=j_{0} \cos (\theta), \\
J_{Q}=j_{0} \sin (\theta) \cos (\psi), \\
\dot{\varphi}\left(k_{B} T\right)=j_{0} \sin (\theta) \sin (\psi),
\end{gathered}
$$

where $j_{0}=\sqrt{J_{c}^{2}\left(k_{B} T\right)^{2} / e^{2}+J_{Q}^{2}+\dot{\varphi}^{2}\left(k_{B} T\right)^{2}}$. The figure of merit at given $\theta$ and $\psi$ is given by

$$
\xi(\theta, \psi)=\frac{\lambda(\theta, \psi)}{1-\lambda(\theta, \psi)}, \lambda(\theta, \psi)=\frac{\lambda_{1}}{1+2 \lambda_{2}},
$$

with

$$
\begin{aligned}
\lambda_{1}= & {\left[q_{c w} \cos (\theta)+q_{Q_{w}} \sin (\theta) \cos (\psi)+q_{c w} \sin (\theta) \sin (\psi)\right] } \\
\lambda_{2}= & {\left[q_{c Q} \cos (\psi)+q_{c \psi} \sin (\psi)\right] \sin (\theta) \cos (\theta)+} \\
& 2 q_{c \psi} \sin ^{2}(\theta) \sin (\psi) \cos (\psi)
\end{aligned}
$$
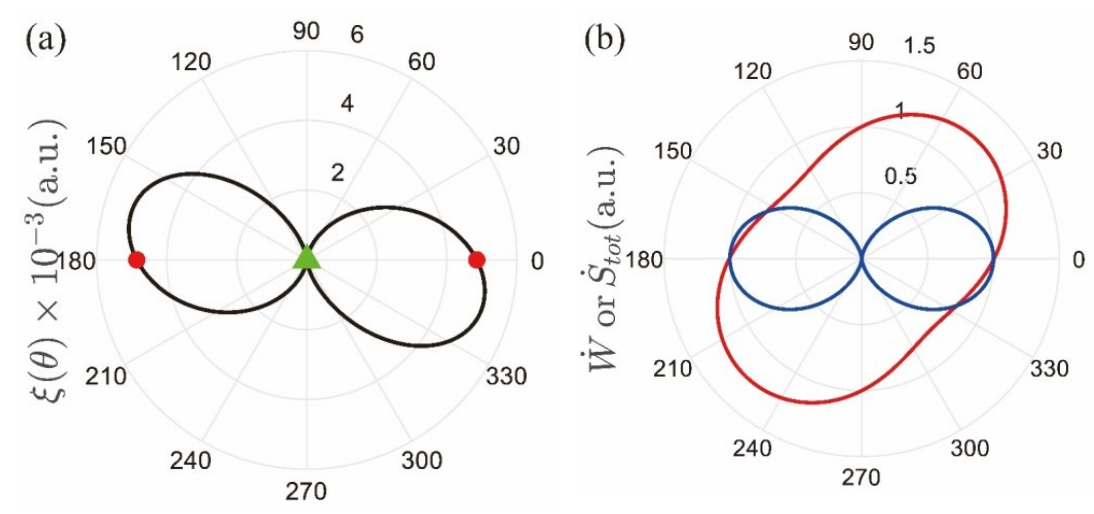

Fig. 6 (a) Polar plot of $\xi$ as the function of $\theta$ for movingthe magnetic domain wall with both electrical and heat currents. $\theta$ is introduced in Eq. (43). The red dots represent thefigure of merit for moving magnetic domain by the electricalcurrent, while the green triangle represents the figure of meritfor moving magnetic domain by the heat current (which is, infact, zero). (b) Polar plot of the output work $\dot{W}$ (blue curve)and the total entropy production $\dot{S}_{\text {tot }}$ (red curve) as functionsof $\theta$ for the same system. 

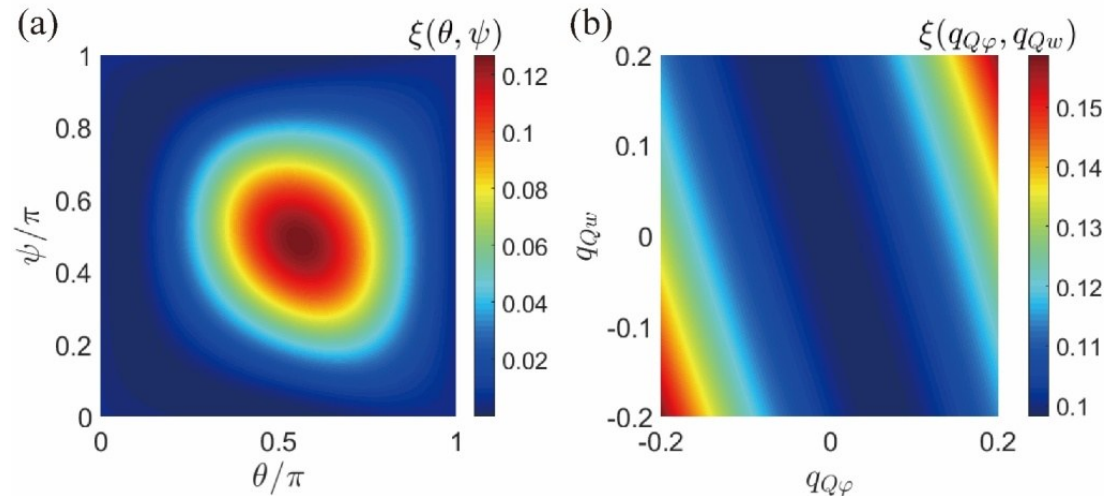

Fig. 7 (a) The figure of merit $\xi(\theta, \psi)$ for moving magneticdomain using electrical, heat, mechanical motions in a magnetic wire. Parameters are given in the main text. (b) Thefigure of merit $\xi\left(q_{Q_{w}}, q_{Q \varphi}\right)$ for moving magnetic domain using electrical, heat, mechanical motions in a magnetic wire.Parameters are given in the main text.

The figure of merit $\xi(\theta, \psi)$ is plotted in Fig. 7(a). The figure of merit is maximized near $\theta \approx \pi / 2$ and $\psi \approx \pi / 2$ because the coupling between domain motion and mechanical motion is the strongest one. Nevertheless, including the electrical and heat currents still improves the figure of merit. The maximum figure of merit due to cooperative effect when considering moving magnetic domain by electrical, thermal, and mechanical currents is given by Eq. (15). We calculate the figure of merit for finite $q_{Q_{w}}$ and $q_{Q_{\varphi}}$ to check how thermal-domain-motion coupling and thermal-mechanical coupling affect the figure of merit. The results are plotted in Fig. 7(b). Indeed the figure of merit is considerably improved by finite $q_{Q_{w}}$ and $q_{Q_{\varphi}}$.

\section{Conclusion and Discussions}

We have shown that cooperative effects can be a potentially useful tool in improving the energy efficiency of spin caloritronic devices. For example, the cooperative effect can greatly enhance the figure of merit, if the magnetic DW motion is driven by the electrical and heat currents concurrently. Our scheme provides a new route to significantly enhance the energy efficiency and hence considerably reduce Joule heating for future advanced information storage and information processing based on magnetic materials.

\section{Acknowledgments}

J. L. and J. H. J. acknowledge support from the National Natural Science Foundation of China (NSFC Grant No. 11675116), the Jiangsu distinguished professor funding and a Project Funded by the Priority Academic Program Development of Jiangsu Higher Education Institutions (PAPD). He thanks Prof. Gerrit E. W. Bauer for many helpful and stimulating discussions. F. Z and Z. Z. S acknowledge supports from the National Natural Science Foundation of China (NSFC Grant No. 11274236) and Research Fund for the Doctoral Program of Higher Education of China (RFDP Grant No. 20123201110003).

\section{Appendix A: Thermodynamic Bound on the Coefficients of Spin-Thermoelectric Transport}

Spin-thermoelectric transport is described by phenomenological equation

$$
\left(\begin{array}{l}
J_{c} \\
J_{s} \\
J_{Q}
\end{array}\right)=\left(\begin{array}{ccc}
G & G P & G S T \\
G P & G & G S^{\prime} T \\
G S T & G S^{\prime} T & K_{0} T
\end{array}\right)\left(\begin{array}{c}
\Delta V \\
\Delta m \\
\Delta T / T
\end{array}\right) .
$$

Due to the second law of thermodynamics, the transport coefficients are bounded by the following inequalities (keep in mind that $G=\sigma A / l$ and $K_{0}=\kappa_{0} A / l$ ),

$$
P^{2} \leqslant 1, \sigma S^{2} T \leqslant \kappa_{0}, \sigma T S^{2} \leqslant \kappa_{0},
$$

as well as that the determinant of the Onsager matrix is non-negative,

$$
\kappa_{0}\left(1-P^{2}\right)-\sigma T\left(S^{2}-2 P S S^{\prime}+S^{2}\right) \geqslant 0 .
$$

Using the relations

$$
\begin{gathered}
\sigma=\int d E\left(-\frac{\partial n_{F}}{\partial E}\right) \sum_{s} \sigma^{(s)}(E), \\
P=\left\langle s_{z}\right\rangle, \quad S^{\prime}=\frac{\left\langle E s_{z}\right\rangle}{e T}, \\
S=\frac{\langle E\rangle}{e T}, \quad \kappa_{0} T=e^{-2} \sigma\left\langle E^{2}\right\rangle
\end{gathered}
$$

the inequalities in (A2) can be rewritten as

$$
-1 \leqslant\left\langle s_{z}\right\rangle \leqslant 1,\langle E\rangle^{2} \leqslant\left\langle E^{2}\right\rangle,\left\langle E s_{z}\right\rangle^{2} \leqslant\left\langle E^{2}\right\rangle .
$$


The first two are obvious. The last inequality in the above is guaranteed by the Cauchy-Schwarz inequality $\langle X Y\rangle^{2} \leqslant\left\langle X^{2}\right\rangle\left\langle Y^{2}\right\rangle$ since $\left\langle s_{z}^{2}\right\rangle=1$ (keep in mind that $s_{z}$ takes the discrete values of 1 for spin up and -1 for spin down). The inequality (A3) is guaranteed by theCauchy-Schwarz inequality

$$
\left|\operatorname{Cov}\left(E, s_{z}\right)\right|^{2} \leqslant \operatorname{Var}(E) \operatorname{Var}\left(s_{z}\right) .
$$

Specifically,

$$
\begin{gathered}
\left|\operatorname{Cov}\left(E, S_{z}\right)\right|^{2}=\left(\left\langle E s_{z}\right\rangle-\langle E\rangle\left\langle S_{z}\right\rangle\right)^{2}= \\
e^{2} T^{2}\left[P^{2} S^{2}+S^{2}-2 P S S^{\prime}\right], \\
\operatorname{Var}(E) \operatorname{Var}\left(s_{z}\right)=\left(\left\langle E^{2}\right\rangle-\langle E\rangle^{2}\right)\left(1-\left\langle s_{z}\right\rangle^{2}\right)= \\
\frac{e^{2} T}{\sigma}\left[\left(\kappa_{0}-\sigma S^{2} T\right)\left(1-P^{2}\right)\right] .
\end{gathered}
$$

\section{Appendix B: Spin-Dependent Seebeck Coefficients}

If the energy-dependence of conductivity is weak and almost linear around the chemical potential, i. e., when the energy dependent conductivity can be described by $\sigma^{(s)}(E)=$

$$
\begin{aligned}
\sigma^{(s)}(0)\left[1+E \frac{d \ln \sigma^{(s)}(0)}{d E}\right], \text { we have } \\
P=\frac{\sigma^{(\uparrow)}-\sigma^{(\downarrow)}}{\sigma^{(\uparrow)}+\sigma^{(\downarrow)},} \\
S=\frac{\sigma^{(\uparrow)} S^{(\uparrow)}-\sigma^{(\downarrow)} S^{(\downarrow)}}{\sigma^{(\uparrow)}+\sigma^{(\downarrow)}}, \\
S^{(s)}=\frac{\pi^{2} k_{B}^{2} T}{3 e} \frac{d \ln \sigma^{(s)}(0)}{d E} .
\end{aligned}
$$

We plot the enhancement factor for the figure of merit $\xi / \max \left(\xi_{T E}, \xi_{S P}\right)$ as a function of $S_{\uparrow}$ and $S_{\downarrow}$ for $P=0.5$ in Fig. $S 1(a)$. Strong enhancement of the figure of merit induced by cooperative effect happens when $\left|S_{\downarrow}\right|$ is smaller and $\left|S_{\uparrow}\right|$ is larger (regardless of their signs). For $P=0.5$ and $\kappa_{0}=$ $\sigma L T$, we can get

$$
\begin{gathered}
\xi=\frac{S_{\downarrow}^{2}+3 S_{\uparrow}^{2}}{4 L-S_{\downarrow}^{2}-3 S_{\uparrow}^{2}}, \\
\xi_{T E}=\frac{\left(S_{\downarrow}+3 S_{\uparrow}\right)^{2}}{16 L-\left(S_{\downarrow}+3 S_{\uparrow}\right)^{2}}, \\
\xi_{S P}=\frac{\left(S_{\downarrow}-3 S_{\uparrow}\right)^{2}}{16 L-\left(S_{\downarrow}-3 S_{\uparrow}\right)^{2}} .
\end{gathered}
$$

The factor of 3 appears because $((1+\mathrm{P})) /((1-$ $P))=3$ for $P=0.5$. From the above, the thermoelectric figure of merit $\xi_{T E}$ vanishes when $S_{\downarrow}=-3 S_{\uparrow}$ in Fig. 8(c), while the spin-Peltier cooling figure of merit $\xi_{S P}$ vanishes when $S_{\downarrow}=3 S_{\uparrow}$ in Fig. 8(d). In contrast, the cooperative figure of merit $\xi$ vanishes only when both $S_{\uparrow}$ and $S_{\downarrow}$ are zero in Fig. 8(b). When $\left|S_{\downarrow}\right|$ and $\left|S_{\uparrow}\right|$ are larger (regardless of their signs), strong enhancement of figure of merit by cooperative effect can be achieved. A realistic regime is when $S_{\uparrow}$ and $S_{\downarrow}$ have the same sign. This can be realized in materials where, e.g., the energy dependence of the density of states is much stronger for carriers with the minority spin than for carriers with the majority spin.
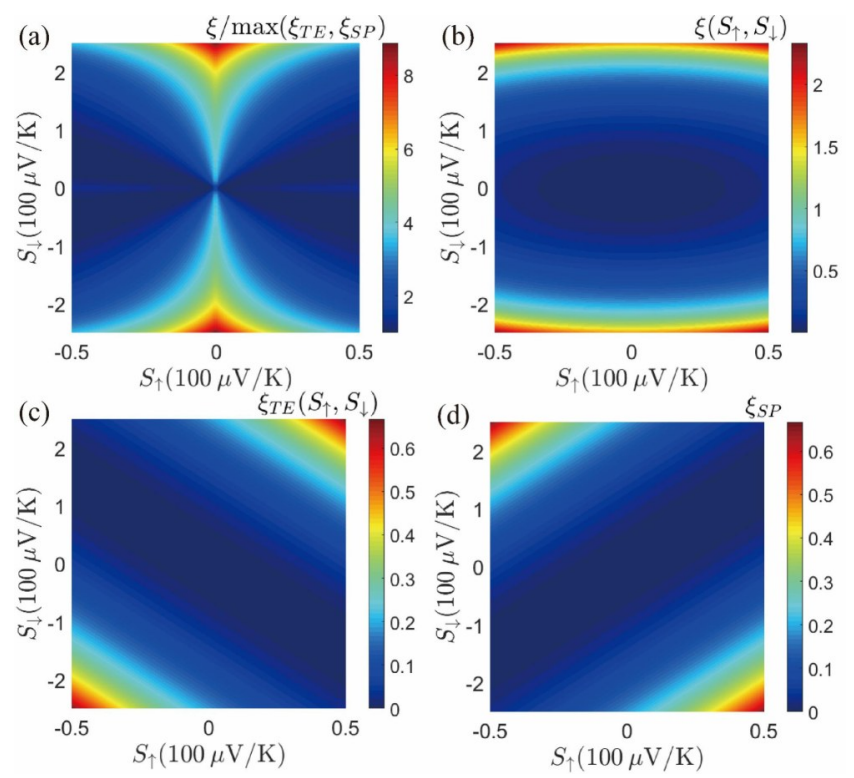

Fig. 8 (Color online) Spin-thermoelectric refrigeration. (a) The enhancement factor, $\frac{\xi}{\max \left(\xi_{T E}, \xi_{S P}\right)}$ as a function of $S_{\uparrow}$ and $S_{\downarrow}$. The parameters are $P=0.5, T=300 \mathrm{~K}, \kappa_{0}=\sigma L T$ with the Lorenz number of $L=2.5 \times 10^{-8} \mathrm{~W} \Omega \mathrm{K}^{-2}$. The figures of merit $\xi(\mathrm{b}), \xi_{T E}(\mathrm{c})$, and $\xi_{S P}$ (d) as functions of $S$, and $S_{\downarrow}$.

\section{Appendix C: The Enhancement of the Figure of Meritdue to Cooperative Effect}

In this section, we prove that the cooperative figure of merit $\xi$, Eq. (19), is always larger than (or, at least, equal to) the figures of merit for energy conversion driven by only one input force.

The dimensionless Onsager matrix has the form

$$
\hat{M}^{\prime}=\left(\begin{array}{ccc}
1 & q_{12} & q_{13} \\
q_{12} & 1 & q_{23} \\
q_{13} & q_{23} & 1
\end{array}\right) .
$$

According to the second law of thermodynamics, we have $\operatorname{det}\left(\hat{M}^{\prime}\right) \geqslant 0$, i.e., $1-q_{12}^{2}-q_{13}^{2}-q_{23}^{2}+2 q_{12} q_{13} q_{23} \geqslant 0$. Let us first prove $\xi \geqslant \xi_{12}$. The figure of merit $\xi_{12}$ is 


$$
\xi_{12}=\frac{1}{1-q_{12}^{2}}-1
$$

Hence, we need to prove $\frac{1-q_{23}^{2}}{1-q_{12}^{2}-q_{13}^{2}-q_{23}^{2}+2 q_{12} q_{13} q_{23}}-$ $\frac{1}{1-q_{12}^{2}} \geqslant 0$, i.e.

$$
q_{13}^{2}-2 q_{12} q_{13} q_{23}+q_{23}^{2} q_{12}^{2} \geqslant 0 .
$$

The above inequality is always true. The left hand side is zero only when $q_{13}=q_{12} q_{23}$. Similarly one can prove that $\xi \geqslant$ $\xi_{13}$. The equality of $\xi$ and $\xi_{13}$ holds only when $q_{12}=q_{13} q_{23}$. Therefore, $\xi$ is always greater than $\xi_{12}$ and $\xi_{13}$, unless $q_{23}=$ $q_{12} / q_{13}$ or $q_{23}=q_{13 /} q_{12}$. Since $\left|q_{13}\right|,\left|q_{12}\right|$ and $\left|q_{23}\right|$ are smaller than 1 , only one of those two conditions can be met for a given physical system.

\section{References}

1. D. A. Allwood, G. Xiong, C. Faulkner, D. Atkinson, D. Petit and R. Cowburn, Science, 2005, 309, 1688.

2. R. P. Cowburn, Nature, 2007, 448, 544.

3. D. Atkinson, D. A. Allwood, G. Xiong, M. D. Cooke, C. C. Faulkner and R. P. Cowburn, Nat. Mater., 2003, 2, 85.

4. S. S. Parkin, M. Hayashi and L. Thomas, Science, 2008, 320, 190.

5. N. Romming, C. Hanneken, M. Menzel, J. E. Bickel, B. Wolter, K. von Bergmann, A. Kubetzka and R. Wiesendanger, Science, 2013, 341, 636.

6. H. Thierschmann, R. Sánchez, B. Sothmann, F. Arnold, C. Heyn, W. Hansen, H. Buhmann and L. W. Molenkamp, Nat. Nanotech., 2015, 10, 854.

7. J. Slonczewski, J. Magn. Magn. Mater., 1996, 159, L1.

8. L. Berger, Phys. Rev. B, 1996, 54, 9353.

9. Z. Li and S. Zhang, Phys. Rev. Lett., 2004, 92, 207203.

10. G. Tatara and H. Kohno, Phys. Rev. Lett., 2004, 92, 086601.

11. G. Beach, C. Knutson, C. Nistor, M. Tsoi and J. Erskine, Phys. Rev. Lett., 2006, 97, 057203.

12. Z. Z. Sun and J. Schliemann, Phys. Rev. Lett., 2010, 104, 037206.

13. S. Emori, U. Bauer, S. M. Ahn, E. Martinez and G. S. Beach, Nat. Mater., 2013, 12, 611.

14. K. S. Ryu, L. Thomas, S. H. Yang and S. Parkin, Nat. Nanotechnol., 2013, $8,527$.

15. J. Iwasaki, M. Mochizuki and N. Nagaosa, Nat. Commun., 2013, 4, 1463.

16. J. Iwasaki, M. Mochizuki and N. Nagaosa, Nat. Nanotechnol., 2013, 8, 742.

17. N. Nagaosa and Y. Tokura, Nat. Nanotechnol., 2013, 8, 899.

18. L. Thomas, R. Moriya, C. Rettner and S. S. Parkin, Science, 2010, 330, 1810.

19. M. Hatami, G. E. W. Bauer, Q. Zhang and P. J. Kelly, Phys. Rev. Lett., 2007, 99, 066603.

20. A. A. Kovalev and Y. Tserkovnyak, Phys. Rev. B, 2009, 80, 100408.

21. X. S. Wang and X. R. Wang, Phys. Rer. B, 2014, 90, 184415.

22. X. S. Wang and X. R. Wang, Phys. Rev. B, 2014, 90, 014414.

23. P. Yan, Y. Cao and J. Sinova, Phys. Rev. B, 2015, 92, 100408.

24. G. Tatara, Phys. Rev. B, 2015, 92, 064405.

25. L. Kong and J. Zang, Phys. Rev. Lett., 2013, 111, 067203.

26. A. A. Kovalev, Phys. Rev. B, 2014, 89, 241101.

27. G. E. Bauer, E. Saitoh and B. J. Van Wees, Nat. Mater., 2012, 11, 391.

28. R. L. Stamps, S. Breitkreutz, J. Akerman, A. V. Chumak, Y. Otani, G. E.
Bauer, J. U. Thiele, M. Bowen, S. A. Majetich and M. Kläui, J. Phys. D: Appl. Phys., 2014, 47, 333001.

29. A. Hoffmann and S. D. Bader, Phys. Rev. Applied, 2015, 4, 047001.

30. C. Z. Fan, Y. Gao and J. P. Huang, Appl. Phys. Lett., 2008, 92, 251907.

31. A. A. Kovalev and Y. Tserkovnyak, Europhys. Lett., 2012, 97, 67002.

32. K. Shen and G. E. W. Bauer, Phys. Rev. Lett., 2015, 115, 197201.

33. I. Juarez-Acosta, M. A. Olivares-Robles, S. Bosu, Y. Sakuraba, T. Kubota, S. Takahashi, K. Takanashi and G. E. W. Bauer, J. Appl. Phys., 2016, 119, 073906.

34. C. Safranski, I. Barsukov, H. K. Lee, T. Schneider, A. Jara, A. Smith, H. Chang, K. Lenz, J. Lindner and Y. Tserkovnyak, Nat. Commun., 2017, 8, 117.

35. A. B. Cahaya, O. A. Tretiakov and G. Bauer, IEEE Trans. Magn., 2015, 51, 1.

36. A. B. Cahaya, O. A. Tretiakov and G. E. W. Bauer, Appl. Phys. Lett., 2014, 104, 042402.

37. A. Kirihara, Nat. Mater., 2012, 11, 686.

38. J. Brüggemann, S. Weiss, P. Nalbach and M. Thorwart, Phys. Rev. Lett., 2014, 113, 076602.

39. N. N. Mojumder, D. W. Abraham, K. Roy and D. Worledge, IEEE Trans. Magn., 2012, 48, 2016.

40. X. Q. Yu, Z. G. Zhu, G. Su and A. P. Jauho, Phys. Rev. Applied, 2017, 8, 054038 .

41. J. H. Jiang, Phys. Rev. E, 2014, 90, 042126.

42. L. Onsager, Phys. Rev., 1931, 37, 405.

43. L. Onsager, Phys. Rev., 1931, 38, 2265.

44. P. Pietzonka and U. Seifert, Phys. Rev. Lett., 2018, 120, 190602.

45. J. H. Jiang, B. K. Agarwalla and D. Segal, Phys. Rev. Lett., 2015, 115, 040601.

46. K. Proesmans, B. Cleuren and C. Van den Broeck, Phys. Rev. Lett., 2016, 116, 220601.

47. J. H. Jiang and Y. Imry, C. R. Phys., 2016, 17, 1047.

48. J. Lu, Y. Liu, R. Wang, C. Wang and J. H. Jiang, Phys. Rev. B, 2019, 100, 115438.

49. G. Benenti, G. Casati, K. Saito and R. Whitney, Phys. Rep., 2017, 694, 1.

50. G. Nicolis, Rep. Prog. Phys., 1979, 42, 225.

51. A. De Vos, J. Phys. Chem., 1991, 95, 4534.

52. J. H. Jiang, O. Entin-Wohlman and Y. Imry, Phys. Rev. B, 2012, 85, 075412.

53. J. H. Jiang, O. Entin-Wohlman and Y. Imry, Phys. Rev. B, 2013, 87, 205420.

54. J. H. Jiang, O. Entin-Wohlman and Y. Imry, New J. Phys., 2013, 15, 075021.

55. O. Entin-Wohlman, J. H. Jiang and Y. Imry, Phys. Rer. E, 2014, 89, 012123.

56. L. Li and J. H. Jiang, Sci. Rep., 2016, 6, 31974.

57. J. H. Jiang and Y. Imry, Phys. Rev. Applied, 2017, 7, 064001.

58. R. Wang, J. Lu, C. Wang and J. H. Jiang, Sci. Rep., 2018, 8, 2607.

59. J. H. Jiang and Y. Imry, Phys. Rev. B, 2018, 97, 125422.

60. J. Lu, R. Wang, J. Ren, M. Kulkarni and J. H. Jiang, Phys. Rev. B, 2019, 99, 035129.

61. J. H. Jiang, J. Appl. Phys., 2014, 116, 194303.

62. J. Lu, R. Wang, Y. Liu and J. H. Jiang, J. Appl. Phys., 2017, 122, 044301.

63. G. E. W. Bauer, S. Bretzel, A. Brataas and Y. Tserkovnyak, Phys. Rer. B, 2010, 81, 024427.

64. L. Landau and E. M. Lifshitz, "Statistical physics,"1980.

Publisher's Note Engineered Science Publisher remains neutral with regard to jurisdictional claims in published maps and institutional affiliations. 